Delft University of Technology

\title{
Transition from Strong to Weak Electronic Coupling in a Single-Molecule Junction
}

Frisenda, R.; Van Der Zant, H. S.J.

DOI

10.1103/PhysRevLett.117.126804

Publication date

2016

Document Version

Final published version

Published in

Physical Review Letters

\section{Citation (APA)}

Frisenda, R., \& Van Der Zant, H. S. J. (2016). Transition from Strong to Weak Electronic Coupling in a Single-Molecule Junction. Physical Review Letters, 117(12), [126804].

https://doi.org/10.1103/PhysRevLett.117.126804

\section{Important note}

To cite this publication, please use the final published version (if applicable).

Please check the document version above.

\section{Copyright}

Other than for strictly personal use, it is not permitted to download, forward or distribute the text or part of it, without the consent of the author(s) and/or copyright holder(s), unless the work is under an open content license such as Creative Commons.

\section{Takedown policy}

Please contact us and provide details if you believe this document breaches copyrights.

We will remove access to the work immediately and investigate your claim. 


\title{
Transition from Strong to Weak Electronic Coupling in a Single-Molecule Junction
}

\author{
R. Frisenda* and H. S. J. van der Zant \\ Kavli Institute of Nanoscience, Delft University of Technology, 2600 GA, The Netherlands
}

(Received 26 October 2015; published 16 September 2016)

\begin{abstract}
We have investigated charge transport in single-molecule junctions using gold nanoelectrodes at room and cryogenic $(10 \mathrm{~K})$ temperatures. A statistical analysis of the low-bias conductance, measured during the stretching of the molecular junctions, shows that the most probable single-molecule conductance is insensitive to the temperature as expected for off-resonant coherent transport. Low-temperature currentvoltage measurements show that these junction conformations have a smooth tunnelinglike shape. While separating the electrodes further we find that, in about one-fourth of the cases, the junction switches in an abrupt way to a configuration with $I-V$ characteristics exhibiting a gap around zero bias and resonances at finite bias. The analysis of the $I-V$ shape and of the conductance distance dependence suggests a stretchinginduced transition from the strong to the weak electronic coupling regime. The transition involves a large renormalization of the injection barrier and of the electronic coupling between the molecule and the electrodes.
\end{abstract}

DOI: 10.1103/PhysRevLett.117.126804

Molecular-scale electronics focuses on the investigation of charge transport through individual molecules. Experimentally, the question of what is the electrical conduction through a molecule can be addressed thanks to the development of methods to physically and electrically contact single molecules, such as the break-junction (BJ) techniques $[1,2]$. From a theoretical point of view, different models have been developed to describe transport through a molecule either strongly or weakly attached to electron reservoirs; a unified description capable of describing both regimes, however, is still missing $[3,4]$.

In the strong coupling regime a mean-field description of the electronic correlation is usually employed, while in the weak coupling regime electron-electron interactions need to be considered. Next to the level alignment or injection barrier, $\epsilon_{0}$, charge transport in a single-molecule junction at low temperature is determined by the electronic coupling to the metallic electrodes, $\Gamma$, and the charging energy, $U$, that describes the electrostatic energetic cost of placing an electron on the molecule and is related to electron-electron interactions. The total electronic coupling $\Gamma$ is the sum of the individual coupling of the molecule to the left and to the right electrodes, $\Gamma=\Gamma_{L}+\Gamma_{R}$. It is known that the relative size of $\Gamma$ and $U$ determines the transport regime through the system $[3,4]$. When $U / \Gamma \ll 1$ the system is in the strong coupling regime and transport is mainly due to coherent tunneling while in the opposite limit, with $U / \Gamma \gg 1$, the system is in the weak coupling regime and transport proceeds mostly via sequential tunneling [5,6]. Among single-molecule transport experiments the strong coupling regime is more common [2,7], while the weak coupling regime remains less understood and fewer examples are present in the literature. For example, Danilov et al. [8] showed that the addition of one methylene unit at each end of a linearly conjugated oligo(phenylene-vynilene) dithiol (OPV3) molecule, changes the transport mechanism from coherent with a finite zero-bias conductance to incoherent with Coulomb blockade behavior. In the field of mesoscopic physics the transition from strong to weak electronic coupling has been observed in transport through inorganic quantum dots with electrically tunable tunneling barriers [9] and in carbon nanotubes [10-12].

In this Letter we report on the observation of a transition from strong to weak electronic coupling in a single-molecule junction created with the mechanically controlled breakjunction (MCBJ) technique. We study oligo(phenyleneethynilene) (OPE3) molecules [13-21] terminated with acetyl-protected thiol anchoring groups for $\mathrm{Au}$ nanoelectrodes [22-24]. We perform low-bias conductance measurements of the OPE3 molecules at room $(T \sim 300 \mathrm{~K})$ and at cryogenic temperatures $(T \sim 10 \mathrm{~K})$. From current-voltage characteristics measured at $10 \mathrm{~K}$ we find that most of the junctions have $I-V$ 's with a smooth tunnelinglike shape typical of coherent transport [25-27], that persists for the full stretching of the junction until the final rupture is reached. In contrast, $27 \%$ of the molecular junctions show an abrupt transition from smooth tunnelinglike $I-V$ 's to $I-V$ 's with a suppression of the current at low bias, typical of weak coupling and Coulomb blockade [28-31], induced by the stretching. The results indicate that when separating the electrodes, the ratio $U / \Gamma$ can change from small to large values, thereby modifying the transport regime and the $I-V$ shape.

Single-molecule junctions are created by repeatedly forming and breaking a $\mathrm{Au}$ wire in the presence of OPE3, shown in Fig. 1(a). We deposit on a MCBJ sample a $2 \mu \mathrm{L}$ droplet of a $1 \mathrm{mmol} / \mathrm{L}$ solution of OPE3, dissolved in dichloromethane (DCM), with the addition of tetra-butyl 

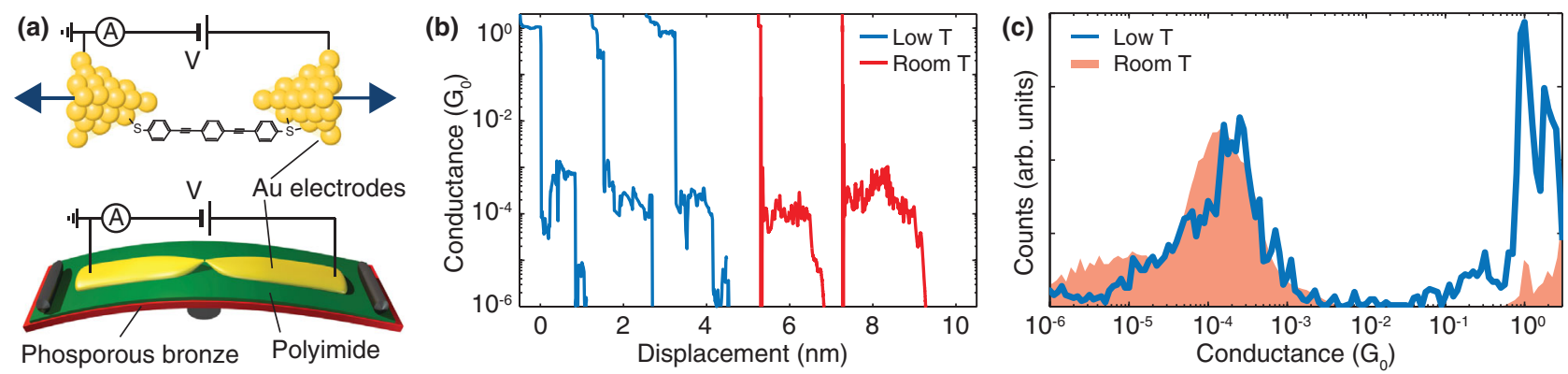

FIG. 1. (a) Schematic illustrations of a Au-molecule-Au junction formed with mechanically controllable break-junction nanoelectrodes (top) and of the sample with the bending mechanism (bottom). (b) Individual conductance versus displacement traces, recorded in the presence of OPE3, measured at a bias of $100 \mathrm{mV}$. The blue curves have been measured near the liquid helium temperature $(10 \mathrm{~K})$ and the red curves at room temperature $(300 \mathrm{~K})$. The traces have been offset along the $x$ axis for clarity. (c) Conductance histogram built from conductance traces that contain molecular junctions at both temperatures.

ammonium hydroxide (TBAH) to promote the hydrolysis of the acetyl-protected thiol groups [23,32]. The process of breaking the Au wire in the presence of OPE3 creates two atomically sharp nanoelectrodes, where the deprotected thiol-terminal groups can bind to form single-molecule junctions.

Figure 1(b) shows low-bias conductance vs distance traces $(G(d))$, measured during the stretching of the electrodes in the presence of OPE3, at room temperature and at $10 \mathrm{~K}$. In the region below $1 G_{0}$, the quantum of conductance $G_{0} \approx 77.5 \mu \mathrm{S}$, the conductance traces show plateaus, typically at $G \approx 10^{-4} G_{0}$, attributed to the formation of single-molecule junctions [33-36]. The plateaus extend for a length of about $1.5 \mathrm{~nm}$ after which the conductance drops to the noise level of the setup $\left(G \simeq 2 \times 10^{-7} G_{0}\right)$. To perform a statistical analysis of the conductance of OPE3 we measured more than 1000 breaking traces at each temperature and we built conductance histograms by selecting the traces that show a conductance plateau in the region between $10^{-2} G_{0}$ and $10^{-5} G_{0}$. The logarithmically binned conductance histograms are compared in Fig. 1(c). The histograms show a clear conductance peak indicating the formation of molecular junctions. We find that the average conductance of OPE3 at room temperature, defined as the maximum of the peak, is $G=1.5 \times 10^{-4} G_{0}$, comparable to the value found in previous studies [16-21,37], and that the peak width corresponds to 1 order of magnitude. The conductance histogram, built from low temperature measurements, shows a similar average conductance value and peak width.

At low temperature we have recorded $I-V$ 's at different displacements of the electrodes and Fig. 2(a) plots the lowand high-bias conductance trace of junction 1, built from the $I-V$ 's by performing a linear fit around zero bias and by dividing the current at $0.9 \mathrm{~V}$ by the bias voltage. Both the low- and high-bias conductance traces show a plateau around $G \approx 10^{-4} G_{0}$ and an abrupt drop of the conductance below the noise level $(I<20 \mathrm{pA}$ for a voltage in the range $\pm 1 \mathrm{~V}$ ) at a displacement close to $1.5 \mathrm{~nm}$. It is interesting that the high-bias conductance trace resembles closely the low-bias one. The conductance plateau contains several jumps with a change in conductance up to 1 order of magnitude. Apparently, while separating the electrodes, the molecule can adapt to the increase in gap size by relaxing internal degrees of freedom or by changing the anchoring site on the electrodes, i.e., by jumping from one gold atom to another [38].

Figure 2(b) displays representative $I-V$ 's recorded during the breaking process, measured at the positions indicated by the arrows in Fig. 2(a). The upper curve measured just after the formation of the molecular junction is asymmetric with a rectification ratio of about 5 at $1 \mathrm{~V}$. The two middle $I-V$ 's are almost symmetric and the last $I-V$, measured just before the breaking, is again asymmetric. All these $I-V$ 's show a similar level of current $I \approx 10 \mathrm{nA}$ at $0.5 \mathrm{~V}$. An asymmetric $I-V$ can be explained by a bias-dependent level alignment, which can be caused, for example, by a different binding motif of the molecule to the left and with right electrodes.
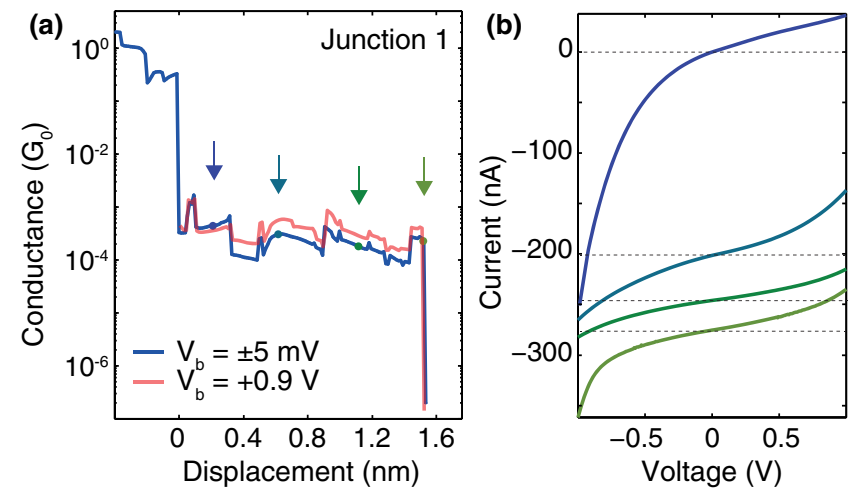

FIG. 2. (a) Low-temperature breaking traces in the presence of OPE3 constructed from $I-V$ 's at low (blue) and high (orange) bias. (b) Current-voltage $(I-V)$ characteristics measured during the breaking event shown in (a). The $I-V$ curves have been shifted along the $y$ axis for clarity. 

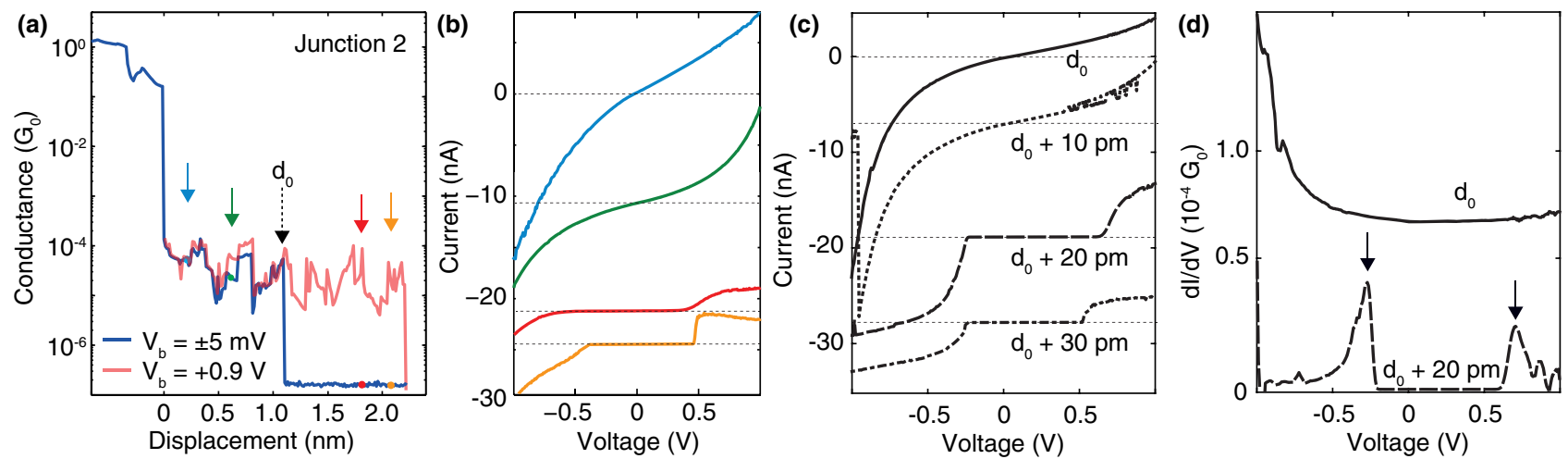

FIG. 3. (a) Low-temperature breaking traces in the presence of OPE3. (b) Current-voltage (I-V) characteristics measured during the breaking event shown in (a). The $I$ - $V$ curves have been shifted along the $y$ axis for clarity. (c) $I$ - $V$ 's measured at four different positions close to the switching point. The $I-V \mathrm{~s}$ are offset on the $y$ axis for clarity. (d) Differential conductance numerically calculated from two $I$ - $V \mathrm{~s}$ in (c). Note that the conductance vs bias curves are not offset, emphasizing the finite conductance of $7 \times 10^{-5} G_{0}$ just before the switching.

Figure 3(a) illustrates the conductance traces measured on junction 2. The low-bias conductance exhibits a plateau around $10^{-4} G_{0}$ that extends for $1.2 \mathrm{~nm}$, similar to the one shown in Fig. 2. At $1.2 \mathrm{~nm}$, the low-bias conductance drops to the noise level, $G \sim 2 \times 10^{-7} G_{0}$, and remains at this value for the subsequent $1 \mathrm{~nm}$ of stretching. In contrast, the high-bias conductance continues to show a plateau around $10^{-4} G_{0}$ up to $2.2 \mathrm{~nm}$ where it drops to the noise level. Figure 3(b) shows that in the first region, up to $1.2 \mathrm{~nm}$ of stretching, the $I-V$ 's are nonlinear with a tunnelinglike shape, comparable to the $I-V$ 's of junction 1 . The $I-V$ 's are asymmetric at the beginning of the conductance plateau and then become more symmetric, as evidenced by the green curve. From $1.2 \mathrm{~nm}$ to the end of the trace, however, the $I$-V's exhibit a different shape, characterized by a gap in the current at low bias [Fig. 3(b); lower two traces]. At a threshold voltage of about $V \approx \pm 0.5 \mathrm{~V}$ the current increases in a step-way fashion. Note that the two bottom $I-V$ 's in Fig. 3(b) present an asymmetry in shape with similar voltage onsets at positive and negative voltages. On the other hand, the two bottom I-V's of Fig. 3(c) have asymmetric voltage onsets; this can be explained by a different voltage drop in the molecule at positive and negative bias, caused for instance by an spatially asymmetric junction configuration or by charging effects (see Refs. [39,40] for a discussion of the possible sources of asymmetry in Coulomb blockade (CB $I-V$ 's).

Figure 3(c) illustrates the transition in more detail by displaying four $I-V$ and $d I / d V-V$ curves measured just before and after the transition point $d_{0}$. Just before the transition the $I-V$ 's appear smooth and featureless, with a conductance at $100 \mathrm{mV}$ of $7 \times 10^{-5} G_{0}$. Increasing the distance by $20 \mathrm{pm}$ results in a dramatic change in the $I-V$ shape; a suppression of the current at low-bias is observed and two distinct resonances appear at $700 \mathrm{mV}$ and $-275 \mathrm{mV}$ [as illustrated in Fig. 3(d)]. While the current and the conductance at low-bias voltages are strongly reduced after the switch, the current and conductance at high bias remain approximately the same, as shown in Figs. 3(a) and 3(c).

To understand the observed change in the shape of the $I-V$ 's while stretching, we start by comparing the roomand low-temperature conductance histograms of Fig. 1. The comparison shows that the average molecular conductance at $10^{-4} G_{0}$ is independent of temperature, a result typical of off-resonant coherent tunneling through the molecule [3,41-43]. Moreover, the $I-V$ 's measured on the plateaus around $10^{-4} G_{0}$ have a smooth tunnelinglike shape, again consistent with coherent transport and strong moleculeelectrode coupling, i.e., $\Gamma>U[25,27]$.

In $27 \%$ of the molecular junctions we observe a stretching-induced low-bias current suppression in the current-voltage curves, as shown for junction 2 in Fig. 3 and for two additional junctions in the Supplemental Material [44]. Comparing different I-V's of junction 2 (see also the Supplemental Material) we see that the gap size depends nonmonotonically on the electrode displacement. Furthermore, the presence in Fig. 3(a) of continuous regions and jumps in the high-bias conductance trace of junction 2 point to a scenario where the molecule is still mechanically connected to both electrodes [45]. From these observations we conclude that the separation of the electrodes induces rearrangements of the molecule in the metallic gap, leading to jumps in the current caused by a change in electronic coupling, a change in level alignment, or a combination of both. On the other hand, if only one electrode were to be mechanically connected to the molecule, one would expect a monotonic decrease in the current as only the weakest molecule-metal coupling would be further diminished. The most likely explanation for the current suppression at low bias is therefore Coulomb blockade $[8,46-48]$ with the molecule mechanically anchored to both electrodes. The question that arises then is, what microscopically triggers the transition from coherent 

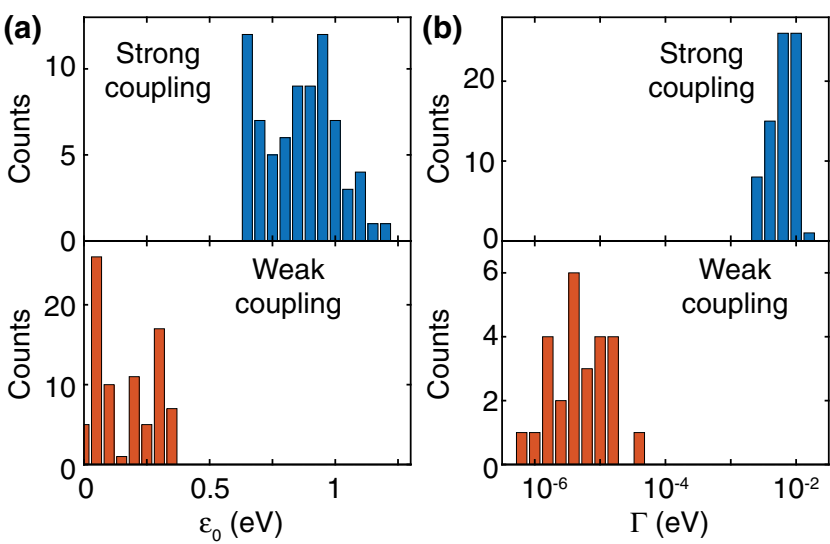

FIG. 4. Histograms of the level alignment (a) and of the electronic coupling (b) for the strongly (top) and weakly (bottom) coupled configuration of junction 2 .

tunneling to $\mathrm{CB}$ transport? or to put in other terms, what induces charge localization on the molecular bridge?

Just after their formation, the molecular junctions have smooth tunnelinglike $I-V$ 's indicating that transport is coherent and the molecule is strongly coupled to the leads $(\Gamma>U)$. By stretching the junctions further, the observed blockaded $I-V$ shape indicates a weak electronic coupling between the molecule leading to Coulomb blockade effects $(\Gamma<U)$. In the $\mathrm{CB}$ regime electron-electron interactions effectively suppress at low-bias transport through the molecule creating a gap in current around zero bias. When a voltage is applied large enough to move a molecular orbital in the bias window, the blockade is lifted and current can flow through the molecule. The voltage needed to include a level in the bias window is 2 times the level alignment since the bias voltage drops across two interfaces between molecule and metal, and thus the voltage gap in the $I-V$ 's is four times the level alignment. The voltage gap size, in the case of symmetric coupling [3,27,49], is related to the level alignment by $e V_{\text {gap }} \approx 4 \epsilon_{0}$, where $e$ is the electron charge and $\epsilon_{0}=\left|E_{F}-\epsilon_{\mathrm{HOMO}}\right|$ or $\epsilon_{0}=\left|E_{F}-\epsilon_{\mathrm{LUMO}}\right|$ depending on whether the HOMO or the LUMO is closest to $E_{F}$, the Fermi energy of the gold leads.

To get more quantitative information from the data, we first consider the level alignment or injection barrier. Before the transition, no resonances are present in the $I-V$ 's within the bias window of $\pm 1 \mathrm{~V}$, indicating that the level alignment is $\epsilon_{0}>0.5 \mathrm{eV}$. We fit the $I-V$ 's in this regime to the Breit-Wigner single-level model for transport and extract the level alignment $\epsilon_{0}$ and the coupling $\Gamma$ (see Supplemental Material [44] for the details of the fit and a similar analysis on two additional junctions). The top panel of Fig. 4(a) shows that the average level alignment is around $0.6-0.9 \mathrm{eV}$, consistent with values previously found at room temperature [20,21]. For the CB $I-V$ 's we extract $\epsilon_{0}$ directly from the position of the step or linear onset in the current $\left(\epsilon_{0}=e V_{\text {gap }} / 4\right)$. The bottom panel of Fig. 4(a) reveals that the level alignment in the $\mathrm{CB}$ regime takes values around $0.1-0.3 \mathrm{eV}$, indicating a smaller injection barrier with respect to the strong coupling regime. We do not include the charging energy in the formula for the determination of the level alignment, since an analytical expression that relates the two quantities does not exist [40]. We notice, however, that by neglecting the charging energy $U$, we are overestimating $\epsilon_{0}$, nevertheless this overestimation would not change the conclusions of the Letter.

The top panel of Fig. 4(b) shows the histogram of the total electronic coupling of junction 2 in the strong coupling regime extracted from the single-level model fit of the $I-V$ 's. The average electronic coupling is about $10 \mathrm{meV}$, again consistent with previous room temperature measurements $[20,21]$. In the case of the CB $I-V$ 's we extract the electronic coupling from the formula $I_{\text {sat }}=\left(G_{0} / e\right) \pi \Gamma$, where $I_{\text {sat }}$ is the saturation current at a bias beyond the resonance $\left(e V \gg 2 \epsilon_{0}+\Gamma\right)$ [3]; the histogram in the bottom panel of Fig. 4(b) displays these values showing electronic coupling around $0.01 \mathrm{meV}$ in this regime. In a many body description the saturation current is influenced both by the total coupling and by the charging energy but no analytical formulas exist for this regime. Thus, the coupling that we find could be underestimated because of charging effects. A deeper theoretical analysis would be beneficial to fit the data to more complicated models, for example by using a selfconsistent formula to include the charging energy $U$ in the fitting procedure. From the values of the coupling found we estimate the charging energy $U \approx 5 \mathrm{meV}$, as discussed in the Supplemental Material [44].

To summarize, we showed that the transition from strong to weak coupling is accompanied by a renormalization of the frontier molecular orbitals' energies together with a strong reduction of the electronic coupling between molecule and electrodes. Similar trends are observed for the other junctions and presented in the Supplemental Material [44] together with the estimation of the charging energy. From the present data we cannot draw a definitive conclusion about the origin of the transition. One possibility is that while stretching, the molecule binds to the apex gold atoms in the final part of the breaking trace. In such a configuration a smaller overlap between the molecule and electrodes may reduce the screening effects in the molecule [50-52]. Consequently, the electron-electron repulsion increases and this could suppress the flow of charges at low bias and drive the transport from coherent to sequential. On the other hand, other possibilities that can rationalize the transition are the formation of a polaron $[53,54]$ or the sudden access to a different charge state of the molecule [55], that due to its electronic structure could be characterized by a different transmission and/or transport mechanism.

In conclusion, the experiment presented here shows an abrupt transition from coherent to sequential tunneling in an OPE3 single-molecule junction. The stretching-induced transition is accompanied by a large renormalization of the 
electronic configuration of the molecule implying that small changes in the junction geometry can have dramatic effects on the transport at the nanoscale. OPE3 provides a model system to study this transition in more detail and theoretical predictions would be helpful to gain further insights into the long-standing problem of the unification of strong and weak coupling regimes into the same theoretical framework.

The authors thank J.M. Thijssen and A. Nitzan for discussions and acknowledge financial support by the Dutch organization for Fundamental Research (FOM), The Netherlands Organisation for Scientific Research (NWO) and by the EU FP7 program through an advanced ERC grant (Mols@Mols).

*Present address: Instituto Madrileño de Estudios Avanzados en Nanociencia (IMDEA-nanociencia), Campus de Cantoblanco, E-28049 Madrid, Spain.

†h.s.j.vanderzant@tudelft.nl

[1] B. Q. Xu and N. J. J. Tao, Science 301, 1221 (2003).

[2] S. V. Aradhya and L. Venkataraman, Nat. Nanotechnol. 8, 399 (2013).

[3] J.C. Cuevas and E. Scheer, Molecular Electronics: An Introduction to Theory and Experiment, Vol. 1 (World Scientific, Singapore, 2010).

[4] D. Supriyo, Nanotechnology 15, S433 (2004).

[5] M. Büttiker, Phys. Rev. B 33, 3020 (1986).

[6] M. Büttiker, IBM J. Res. Dev. 32, 63 (1988).

[7] P. Darancet, J. R. Widawsky, H. J. Choi, L. Venkataraman, and J. B. Neaton, Nano Lett. 12, 6250 (2012).

[8] A. Danilov, S. Kubatkin, S. Kafanov, P. Hedegärd, N. Stuhr-Hansen, K. Moth-Poulsen, and T. Bjørnholm, Nano Lett. 8, 1 (2008).

[9] E. B. Foxman, P. L. McEuen, U. Meirav, N. S. Wingreen, Y. Meir, P. A. Belk, N. R. Belk, M. A. Kastner, and S. J. Wind, Phys. Rev. B 47, 10020 (1993).

[10] B. Babić and C. Schönenberger, Phys. Rev. B 70, 195408 (2004).

[11] J. Cao, Q. Wang, and H. Dai, Nat. Mater. 4, 745 (2005).

[12] A. Makarovski, J. Liu, and G. Finkelstein, Phys. Rev. Lett. 99, 066801 (2007).

[13] Z. J. Donhauser, B. A. Mantooth, K. F. Kelly, L. A. Bumm, J. D. Monnell, J. J. Stapleton, D. W. Price Jr., A. M. Rawlett, D. L. Allara, J. M. Tour, and P. S. Weiss, Science 292, 2303 (2001).

[14] M. Mayor, H. B. Weber, J. Reichert, M. Elbing, C. von Hanisch, D. Beckmann, and M. Fischer, Angew. Chem., Int. Ed. Engl. 42, 5834 (2003).

[15] S. Kubatkin, A. Danilov, M. Hjort, J. Cornil, J. L. Bredas, N. Stuhr-Hansen, P. Hedegärd, and T. Bjørnholm, Nature (London) 425, 698 (2003).

[16] X. Xiao, L. A. Nagahara, A. M. Rawlett, and N. Tao, J. Am. Chem. Soc. 127, 9235 (2005).

[17] S. M. Wu, M. T. Gonzalez, R. Huber, S. Grunder, M. Mayor, C. Schonenberger, and M. Calame, Nat. Nanotechnol. 3, 569 (2008).
[18] R. Huber, M. T. Gonzalez, S. Wu, M. Langer, S. Grunder, V. Horhoiu, M. Mayor, M. R. Bryce, C. S. Wang, R. Jitchati, C. Schönenberger, and M. Calame, J. Am. Chem. Soc. 130, 1080 (2008).

[19] V. Kaliginedi, P. Moreno-Garcia, H. Valkenier, W. J. Hong, V. M. Garcia-Suarez, P. Buiter, J. L. H. Otten, J. C. Hummelen, C. J. Lambert, and T. Wandlowski, J. Am. Chem. Soc. 134, 5262 (2012).

[20] R. Frisenda, M. L. Perrin, H. Valkenier, J. C. Hummelen, and H. S. J. van der Zant, Phys. Status Solidi B 250, 2431 (2013).

[21] R. Frisenda, S. Tarkuç, E. Galan, M. L. Perrin, R. Eelkema, F. C. Grozema, and H. S. J. van der Zant, Beilstein J. Nanotechnol. 6, 1558 (2015).

[22] G. M. Whitesides and P.E. Laibinis, Langmuir 6, 87 (1990).

[23] H. Valkenier, E. H. Huisman, P. A. van Hal, D. M. de Leeuw, R. C. Chiechi, and J. C. Hummelen, J. Am. Chem. Soc. 133, 4930 (2011).

[24] H. Hakkinen, Nat. Chem. 4, 443 (2012).

[25] R. Landauer, IBM J. Res. Dev. 1, 223 (1957).

[26] Y. Imry and R. Landauer, Rev. Mod. Phys. 71, S306 (1999).

[27] S. Lindsay and M. Ratner, Adv. Mater. 19, 23 (2007).

[28] T. A. Fulton and G. J. Dolan, Phys. Rev. Lett. 59, 109 (1987).

[29] C. W. J. Beenakker, Phys. Rev. B 44, 1646 (1991).

[30] A. Troisi and M. Ratner, Small 2, 172 (2006).

[31] S. T. Olsen, V. Arcisauskaite, T. Hansen, J. Kongsted, and K. V. Mikkelsen, Phys. Chem. Chem. Phys. 16, 17473 (2014).

[32] M. T. Gonzalez, E. Leary, R. Garcia, P. Verma, M. A. Herranz, G. Rubio-Bollinger, N. Martin, and N. Agraït, J. Phys. Chem. C 115, 17973 (2011).

[33] H. B. Weber, J. Reichert, F. Weigend, R. Ochs, D. Beckmann, M. Mayor, R. Ahlrichs, and H. v. Löhneysen, Chem. Phys. 281, 113 (2002).

[34] L. Venkataraman, J. E. Klare, C. Nuckolls, M. S. Hybertsen, and M. L. Steigerwald, Nature (London) 442, 904 (2006).

[35] M. T. Gonzalez, S. Wu, R. Huber, S. J. van der Molen, C. Schönenberger, and M. Calame, Nano Lett. 6, 2238 (2006).

[36] F. Chen, X. Li, J. Hihath, Z. Huang, and N. Tao, J. Am. Chem. Soc. 128, 15874 (2006).

[37] Y. J. Xing, T. H. Park, R. Venkatramani, S. Keinan, D. N. Beratan, M. J. Therien, and E. Borguet, J. Am. Chem. Soc. 132, 7946 (2010).

[38] M. Kamenetska, M. Koentopp, A. C. Whalley, Y. S. Park, M. L. Steigerwald, C. Nuckolls, M. S. Hybertsen, and L. Venkataraman, Phys. Rev. Lett. 102, 126803 (2009).

[39] F. Zahid, A. W. Ghosh, M. Paulsson, E. Polizzi, and S. Datta, Phys. Rev. B 70, 245317 (2004).

[40] O. D. Miller, B. Muralidharan, N. Kapur, and A. W. Ghosh, Phys. Rev. B 77, 125427 (2008).

[41] W. Y. Wang, T. Lee, and M. A. Reed, Phys. Rev. B 68, 035416 (2003).

[42] Y. Selzer, L. Cai, M. A. Cabassi, Y. Yao, J. M. Tour, T. S. Mayer, and D. L. Allara, Nano Lett. 5, 61 (2005).

[43] X. L. Li, J. He, J. Hihath, B. Q. Xu, S. M. Lindsay, and N. J. Tao, J. Am. Chem. Soc. 128, 2135 (2006). 
[44] See Supplemental Material at http://link.aps.org/ supplemental/10.1103/PhysRevLett.117.126804 for additional data measured on the MCBJ device studied, which includes Ref. [55].

[45] H. Rascón-Ramos, J. M. Artés, Y. Li, and J. Hihath, Nat. Mater. 14, 517 (2015).

[46] J. Park, A. N. Pasupathy, J. I. Goldsmith, C. Chang, Y. Yaish, J. R. Petta, M. Rinkoski, J. P. Sethna, H. D. Abruna, P. L. McEuen, and D. C. Ralph, Nature (London) 417, 722 (2002).

[47] S. Kubatkin, A. Danilov, M. Hjort, J. Cornil, J.-L. Brédas, N. Stuhr-Hansen, P. Hedegard, and T. Bjørnholm, Curr. Appl. Phys. 4, 554 (2004).

[48] A. R. Champagne, A. N. Pasupathy, and D. C. Ralph, Nano Lett. 5, 305 (2005).
[49] J. M. Thijssen and H. S. J. Van der Zant, Phys. Status Solidi B 245, 1455 (2008).

[50] J. B. Neaton, M. S. Hybertsen, and S. G. Louie, Phys. Rev. Lett. 97, 216405 (2006).

[51] K. J. Franke and J. I. Pascual, J. Phys. Condens. Matter 24, 394002 (2012).

[52] M. Strange and K. S. Thygesen, Phys. Rev. B 86, 195121 (2012).

[53] M. Galperin, M. A. Ratner, and A. Nitzan, Nano Lett. 5, 125 (2005).

[54] M. Galperin, A. Nitzan, and M. A. Ratner, J. Phys. Condens. Matter 20, 374107 (2008).

[55] J. P. Bergfield, G. C. Solomon, C. A. Stafford, and M. A. Ratner, Nano Lett. 11, 2759 (2011). 